University of Nebraska - Lincoln

DigitalCommons@University of Nebraska - Lincoln

\title{
8-2011
}

\section{Ferroelectric Control of Magnetic Anisotropy}

\author{
Abhijit Mardana \\ University of Nebraska-Lincoln \\ Stephen Ducharme \\ University of Nebraska-Lincoln, sducharme1@unl.edu \\ Shireen Adenwalla \\ University of Nebraska-Lincoln, sadenwalla1@unl.edu
}

Follow this and additional works at: https://digitalcommons.unl.edu/physicsducharme

Part of the Physics Commons

Mardana, Abhijit; Ducharme, Stephen; and Adenwalla, Shireen, "Ferroelectric Control of Magnetic Anisotropy" (2011). Stephen Ducharme Publications. 82.

https://digitalcommons.unl.edu/physicsducharme/82

This Article is brought to you for free and open access by the Research Papers in Physics and Astronomy at DigitalCommons@University of Nebraska - Lincoln. It has been accepted for inclusion in Stephen Ducharme Publications by an authorized administrator of DigitalCommons@University of Nebraska - Lincoln. 


\title{
Ferroelectric Control of Magnetic Anisotropy
}

\author{
A. Mardana, Stephen Ducharme, and S. Adenwalla
}

Department of Physics and Astronomy and the Nebraska Center for Materials and Nanoscience, University of Nebraska-Lincoln, Lincoln, Nebraska 68588-0299, USA

Corresponding author - S. Adenwalla, email sadenwalla1@unl.edu

Abstract

We demonstrate unambiguous evidence of the electric field control of magnetic anisotropy in a wedge-shaped Co film of varying thickness. A copolymer ferroelectric of $70 \%$ vinylidene fluoride with $30 \%$ trifluoroethylene, $\mathrm{P}(\mathrm{VDF}-\mathrm{TrFE})$ overlays the Co wedge, providing a large switchable electric field. As the ferroelectric polarization is switched from up to down, the magnetic anisotropy of the Co films changes by as much as $50 \%$. At the lowest Co thickness the magnetic anisotropy switches from out-of-plane to inplane as the ferroelectric polarization changes from up to down, enabling us to rotate the magnetization through a large angle at constant magnetic field merely by switching the ferroelectric polarization. The large mismatch in the stiffness coefficients between the polymer ferroelectric and metallic ferromagnet excludes typical magnetoelectric strain coupling; rather, the magnetic changes arise from the large electric field at the ferroelectric/ferromagnet interface.
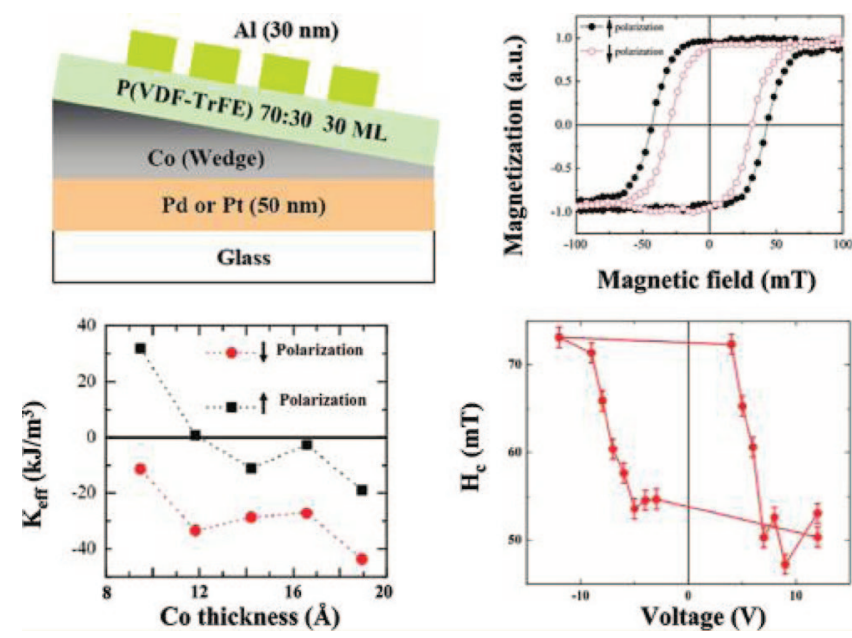

Keywords: Magnetoelectric coupling, electric field control of magnetization, polymer ferroelectric, magnetic anisotropy

$\mathrm{T}_{\mathrm{s}}$ he ability to control magnetic properties with an electric field raises exciting possibilities, both for the understanding of the fundamental physics underlying these effects and for potential technological applications. Electric field control of magnetization has a wide range of applicability in spintronics and magnetic data storage devices, ranging from electrically controllable magnetic memories to magnetoelectric transducers and threshold magnetic sensors. Electric fields inside ferromagnets induce spin-dependent screening charges, ${ }^{1,} 2$ leading to changes in the surface magnetization and surface magnetocrystalline anisotropy. ${ }^{3-6}$ In magnetic semiconductors, the long electric field penetration depths significantly alter the carrier concentration, the Curie temperature, and the saturation magnetization. ${ }^{7,} 8$ Electric field induced changes have also been observed in metallic magnetic thin films, including substantial changes in the coercivity of FePt thin films immersed in a liquid electrolyte, ${ }^{9}$ and changes in the magnetic anisotropy in $\mathrm{Fe} / \mathrm{MgO},{ }^{10} \mathrm{Fe}_{80} \mathrm{Co}_{20} / \mathrm{MgO},{ }^{11}$ and $\mathrm{Co}_{40} \mathrm{Fe}_{40} \mathrm{~B}_{20} / \mathrm{MgO}$ heterostructures. ${ }^{12}$

Ferroelectrics offer a convenient source of large, switchable electric fields, as well as satisfying the need for low power consumption, nonvolatile devices in the realization of electrically controlled magnetic memories. Typically, however, the strain coupling of the magnetic and electrical order parameters $^{13,14}$ in ferroelectric/ferromagnetic heterostructures overwhelms the experimental investigation of more subtle mag- netoelectric effects. ${ }^{15}$ To explore the effects of electric field penetration into a metallic ferromagnet, we fabricated a heterostructure of a stiff metallic ferromagnet, $\mathrm{Co}$, with a soft copolymer ferroelectric, $\mathrm{P}(\mathrm{VDF}-\mathrm{TrFE})$ consisting of $70 \%$ vinylidene fluoride with $30 \%$ trifluoroethylene, with bulk stiffness coefficients on the order of $10^{11}$ and $10^{9} \mathrm{~N} / \mathrm{m}^{2}$, respectively. In general the stiffness coefficients of thin films are expected to differ from those in the bulk by $10-20 \%$, still preserving the large mismatch in stiffness coefficients that will minimize magnetostrictive effects. The soft ferroelectric polymer is unlikely to cause significant strain in the much stiffer metallic Co film. Our experiments provide detailed evidence of the changes in the magnetic anisotropy and free energy of the magnetic film with electric field.

The sample (Figure 1a) consisted of a shallow-angled Co wedge grown on a $\mathrm{Pd}$ or $\mathrm{Pt}$ seed layer with a 30 monolayer film of the ferroelectric copolymer, P(VDF-TrFE) 70:30, deposited by Langmuir-Blodgett deposition ${ }^{16}$ and covered by $\mathrm{Al}$ stripe electrodes at intervals of $3 \mathrm{~mm}$ (Figure $1 \mathrm{~b}$, see the Methods section). The large surface charge density associated with the ferroelectric polarization in $\mathrm{P}(\mathrm{VDF}-\mathrm{TrFE})$, of the order of $0.1 \mathrm{C} / \mathrm{m}^{2}, 17$ is equivalent to an applied field of $1 \mathrm{GV} / \mathrm{m}$, well above the breakdown field of most dielectrics. The anisotropy of the wedge-shaped magnetic film goes from out-of-plane ${ }^{18}$ at the thinnest end to in-plane at the thickest end, undergoing the well-known spin reorientation transition (SRT) at an inter- 

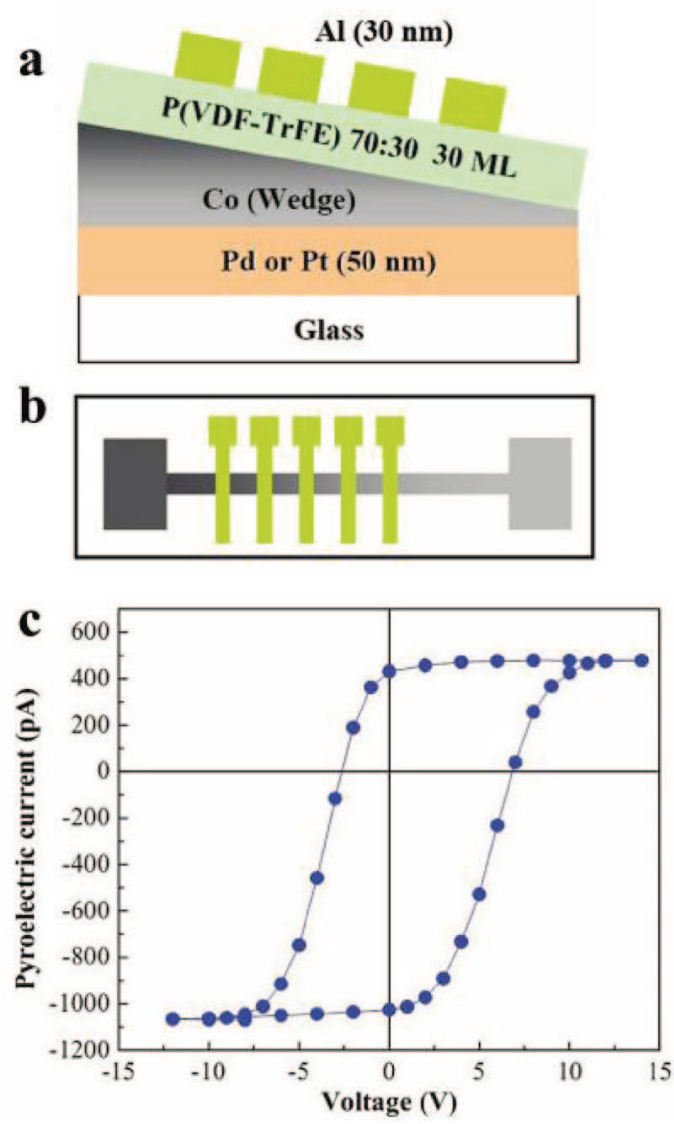
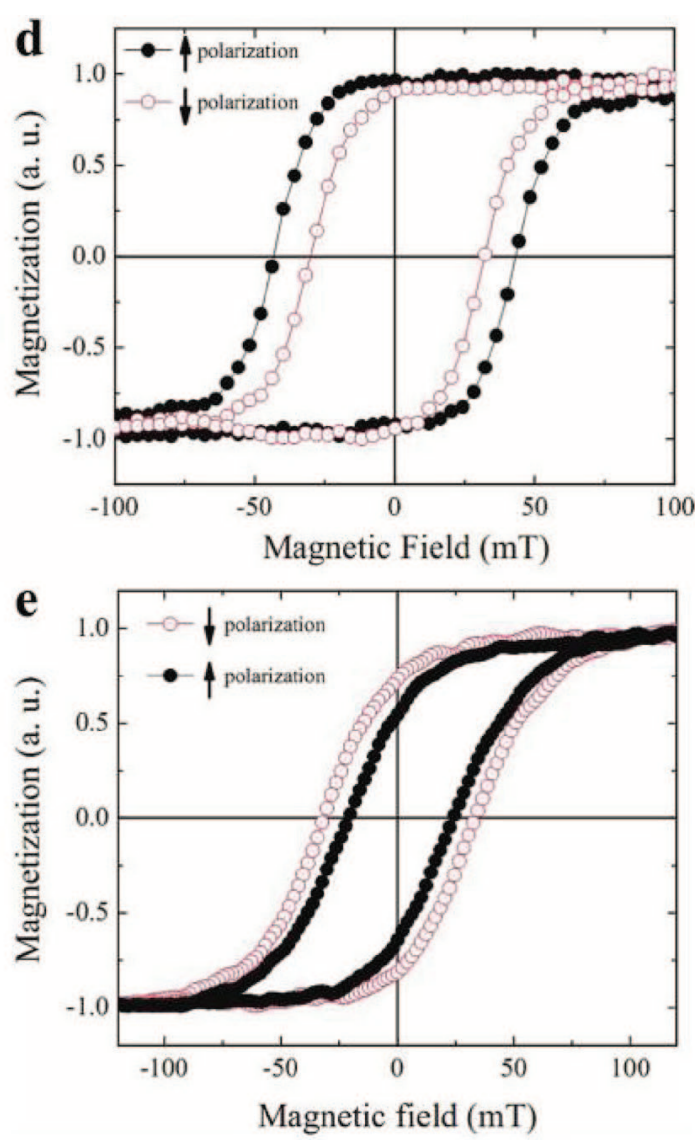

Figure 1. (a) Schematic side view of the sample: glass/(Pd or Pt)/Co (8.5-27.8 $\mathrm{A}) / 30$ monolayers $\mathrm{P}(\mathrm{VDF}-\mathrm{TrFE})$ 70:30/Al(30 nm). The Co strip is $40 \mathrm{~mm}$ long resulting in a very shallow wedge angle of $2.7 \times 10^{-6} \mathrm{deg}$. (b) Schematic diagram of the top view of the sample with bottom (gray) Co electrode and top Al (green) electrode. (c) Ferroelectric hysteresis loop on one particular spot. (d) Out-of-plane hysteresis loops at a Co thickness of 9.5A using PMOKE (polar magneto-optical Kerr effect) depicting the change in coercivity for two different polarization states. Up polarization results in a larger out-of-plane coercivity than down polarization. (e) In-plane magnetization hysteresis loops for the same Co thickness of $9.5 \AA$ using longitudinal MOKE (LMOKE) depicting the changes in coercivity indicate that up polarization (black) results in smaller in-plane coercivity than down polarization (red).

mediate thickness. ${ }^{19}$ The quantity that determines the orientation of the ferromagnetic film is the anisotropy energy, $K_{\text {eff }}$ $=\left(\left(K_{\mathrm{s}} / t\right)-K_{\mathrm{v}}\right)$, where $t$ is the film thickness. The surface anisotropy, $K_{\mathrm{s}^{\prime}}$ favors out-of-plane magnetization and $K_{v^{\prime}}$ the volume term, is dominated by the shape anisotropy, favoring in-plane magnetization. At the SRT, the two energies are comparable, resulting in a very small net anisotropy.

The ferroelectric polarization hysteresis loop (Figure, 1c) demonstrates that voltages of $\pm 12 \mathrm{~V}$ are sufficient to switch the polarization of the ferroelectric film between its two opposing states. ${ }^{20}$ The essence of the magnetoelectric effect is shown in panels d and e of Figure 1, which indicate increased out-ofplane coercivity and decreased in-plane coercivity for up polarization (in which the ferroelectric bound surface charge at the Co surface is negative and the electric field in the Co film points out of the film) vs down polarization. Similar hysteresis loops were measured across the Co wedges down to thicknesses of $9 \AA$, indicating that the Co film is both continuous and ferromagnetic at this thickness.

The influence of the polarization state on the magnetization arises primarily from electric field penetration into the magnetic film. The electric field induces unequal screening for spin-up and spin-down electrons in the ferromagnet changing both the anisotropy energy and the magnetization for the top few atomic layers. ${ }^{2,5}$ We expect the other likely mechanisms of magnetoelectric coupling to be much weaker. Mechanical cou- pling is weak because of the relative softness of the polymer, as noted above. The polymer should have negligible influence on bonding at the metal surface, an expectation supported by first-principles calculations of the interlayer bonding at the $\mathrm{Co} / \mathrm{P}(\mathrm{VDF}-\mathrm{TrFE})$ interface. $^{21}$

The results of both in-plane and out-of-plane magnetization measurements along the Co wedge for both polarization states are summarized in Figure 2, for cobalt films grown on $\mathrm{Pd}$ and Pt seed layers, respectively. These results demonstrate three key factors. (i) The ratio of the remanent magnetization, $M_{r^{\prime}}$ to the saturation magnetization, $M_{\mathrm{s}^{\prime}}$ is shown in panels a and $\mathrm{d}$ of Figure 2 and indicate that Co wedge thickness spanned the spin reorientation transition from out-of-plane to in-plane anisotropy. The position and width of the reorientation transition depend on the underlying seed layer material. (Note that these measurements of the SRT (Figure 2) were made with a virgin ferroelectric film, before the application of an external voltage to polarize the film. However, previous measurements indicate that LB deposition results in films with a preferential up polarization. ${ }^{22}$ Additional experiments described in the Supporting Information show that the presence of the unpoled $\mathrm{P}(\mathrm{VDF}-\mathrm{TrFE})$ film results in a significant shift of the SRT toward the thicker end of the wedge, an effect that may result from subtle chemical changes at the interface.) (ii) When the ferroelectric polarization is pointing away from the metallic Co layer, there is an increase in the out-of-plane coercivity 

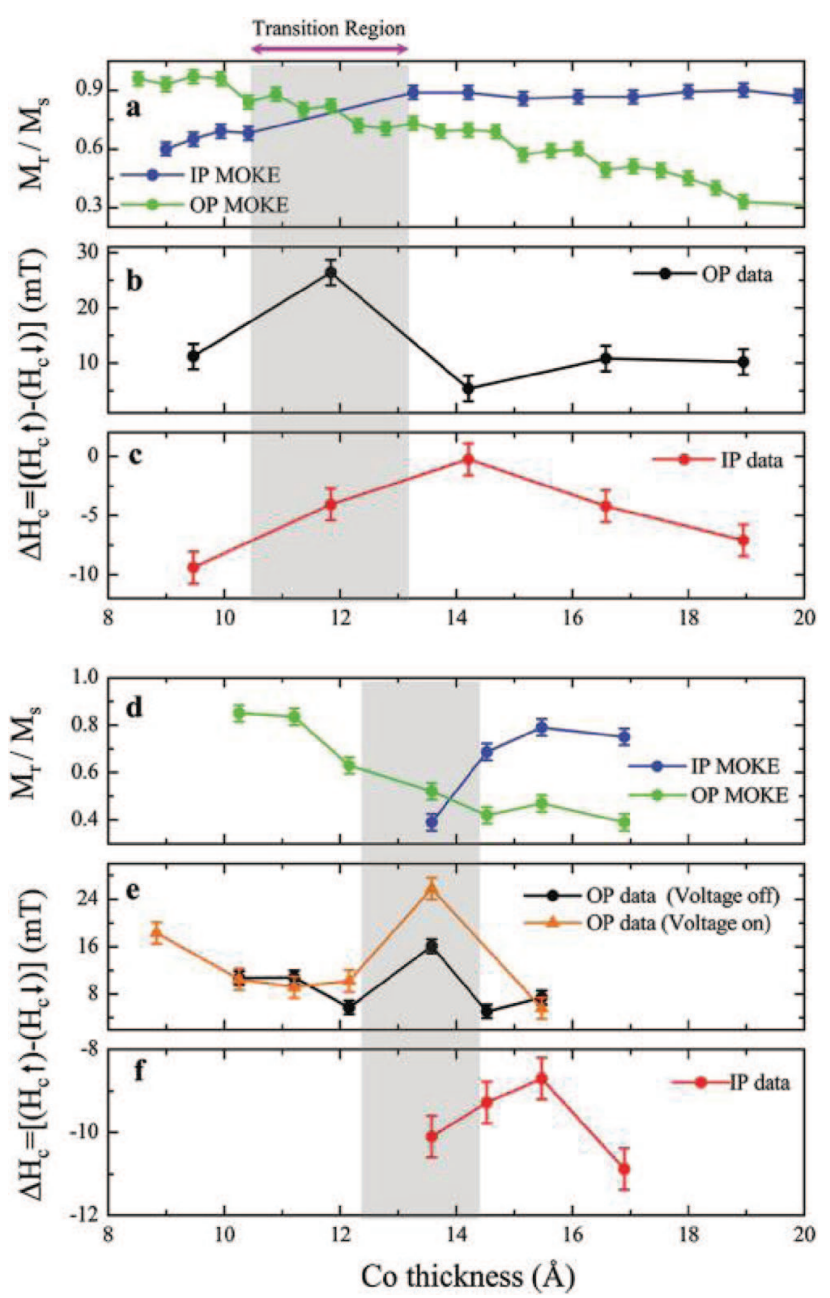

Figure 2. (a), (b), and (c) correspond to the Pd/Co sample and (d), (e), and (f) to the Pt/Co sample. (a) and (d) $M_{\mathrm{r}} / M_{\mathrm{s}}$ data for out-of-plane and in-plane MOKE measurements. $M_{\mathrm{r}} / M_{\mathrm{s}}$ is a measure of the squareness of the magnetization hysteresis loops. Outof-plane and in-plane $M_{\mathrm{r}} / M_{\mathrm{s}}$ measurements indicate the spin reorientation region. Co films grown on both $\mathrm{Pd}$ and $\mathrm{Pt}$ seed layers show a clear spin-reorientation transition region (gray shadow) from out-of-plane to in-plane as the Co thickness increases. (b), (c), (e), and (f) Depicting the difference in coercivity $\Delta H_{\mathrm{c}}=H_{\mathrm{c}}(\mathrm{P} \uparrow)$ $-H_{\mathrm{c}}(\mathrm{P} \downarrow)$, between up and down polarization. (b) and (e) For outof-plane measurements the up polarization state has a larger coercivity than the down polarization state over the entire thickness of Co, with a maximum difference of $26 \mathrm{mT}$ for the $\mathrm{Pd} / \mathrm{Co}$ sample and $16 \mathrm{mT}$ for the $\mathrm{Pt} / \mathrm{Co}$ sample in the SRT region. $\Delta H_{c}$ data with voltage-on are shown for the $\mathrm{Pt} / \mathrm{Co}$ sample (orange). The remaining data are taken at remanence. (c) and (f) In-plane measurements indicate the opposite behavior for the change in coercivity with ferroelectric polarization.

and a decrease in the in-plane coercivity across the entire range of Co thicknesses explored. Clearly, this polarization direction (up) favors out-of-plane anisotropy. Note that for the $\mathrm{Co} / \mathrm{Pt}$ sample, we present results both at ferroelectric remanence, with zero applied voltage, and with an applied voltage of $12 \mathrm{~V}$. The slightly larger changes seen with the voltage on are attributed to the relaxation of the ferroelectric polarization when the voltage is turned off. ${ }^{23}$ (iii) The changes in the out-ofplane coercivity are most pronounced in the region of the spin reorientation transition. In contrast, the changes in the in-plane coercivity are minimized at or close to the SRT.
In contrast to spin-reorientation experiments with $\mathrm{Fe} / \mathrm{MgO}$ thin films, ${ }^{10}$ which require the application of large voltages to perturb the magnetization, the changes seen here are present at zero applied voltage because the remanent ferroelectric polarization produces a large interface charge. This has important ramifications for data storage technology, providing a route to nonvolatile memory storage, because this large remanent polarization is controlled with a relatively small voltage.

To minimize magnetic domain effects in the interpretation of the magnetization data, the effective uniaxial anisotropy constant $K_{\text {eff }}$ for both samples was calculated using the area method. ${ }^{24}$ This method assumes that irreversible domain mechanisms are similar for both in-plane and out-of-plane directions, and hence the difference in area between the out-ofplane and in-plane magnetization curves yields the effective anisotropy energy $K_{\text {eff }}$. (See the Supporting Information for additional details.) As expected from the data in Figure 2a the anisotropy energy for the up polarization state (negative interface charge) changes sign at the SRT (see Figure 3a), going from positive to negative, where a positive value of $K_{\text {eff }}$ corresponds to out-of-plane anisotropy. For the smallest Co thickness measured, switching the ferroelectric polarization to the down state (positive interface charge) alters the uniaxial anisotropy from positive to negative, allowing for electric field controlled switching of the magnetization easy axis from out-ofplane to in-plane.

The reversal of the electrical polarization results in changes in the anisotropy energy, $\Delta K_{\text {eff }}=K_{\text {eff }}(P \uparrow)-K_{\text {eff }}(P \downarrow)$. If we assume that these changes arise solely from the changes in the surface anisotropy energy, we can write $\Delta K_{\mathrm{s}}=t \Delta K_{\text {eff }}$ (where $t$ is the thickness of the Co film). These quantities are shown in panels $c$ and $d$ of Figure 3 as a function of Co thickness and indicate that polarization reversal results in surface anisotropy changes that are in the range of $30-70 \mu \mathrm{J} / \mathrm{m}^{2}$ for both the $\mathrm{Co} / \mathrm{Pd}$ and $\mathrm{Co} / \mathrm{Pt}$ samples. Given the short electric field penetration depth of $\sim 1.5$ $\AA$ in $\mathrm{Co}$, we would expect $\Delta K_{\mathrm{s}}$ to be constant across the whole thickness range. The differences may be due to nonuniform surfaces resulting in variations of the local electric field.

The central role of the switchable polarization of the ferroelectric film, and the resulting electric field in the $\mathrm{Co}$, is further demonstrated by two more experiments. At temperatures above the ferroelectric Curie temperature of the P(VDF-TrFE), where the spontaneous polarization vanishes, the magnetization hysteresis loops showed no change in coercivity (see Supporting Information) because in the paraelectric phase, there was no remanent polarization and no net charge at the interface to influence the magnetic film. Investigations of the magnetic coercivity at intermediate polarization states demonstrated that the magnetic coercive field is proportional to the net ferroelectric polarization, as shown in Figure 4, in which the magnetic coercive fields for out-of-plane hysteresis loops are plotted as a function of the applied electric field, clearly displaying the hysteretic behavior corresponding to the ferroelectric polarization state. The inset of Figure 4 shows the magnetic coercive field plotted as a function of the ferroelectric film polarization (with the relative polarization values obtained empirically from the pyroelectric hysteresis loop), revealing the proportionality between the two, apart from a small remaining hysteresis, which we attribute to incomplete ferroelectric domain switching. The ferromagnetic domains are a few micrometers in size $\mathrm{e}^{25}$ and about 2 orders of magnitude larger than the ferroelectric domains in P(VDF-TrFE), which are $30-50 \mathrm{~nm}$ in size. ${ }^{26}$ Hence, each ferromagnetic domain experiences an electric field that results from the average macroscopic polarization. 

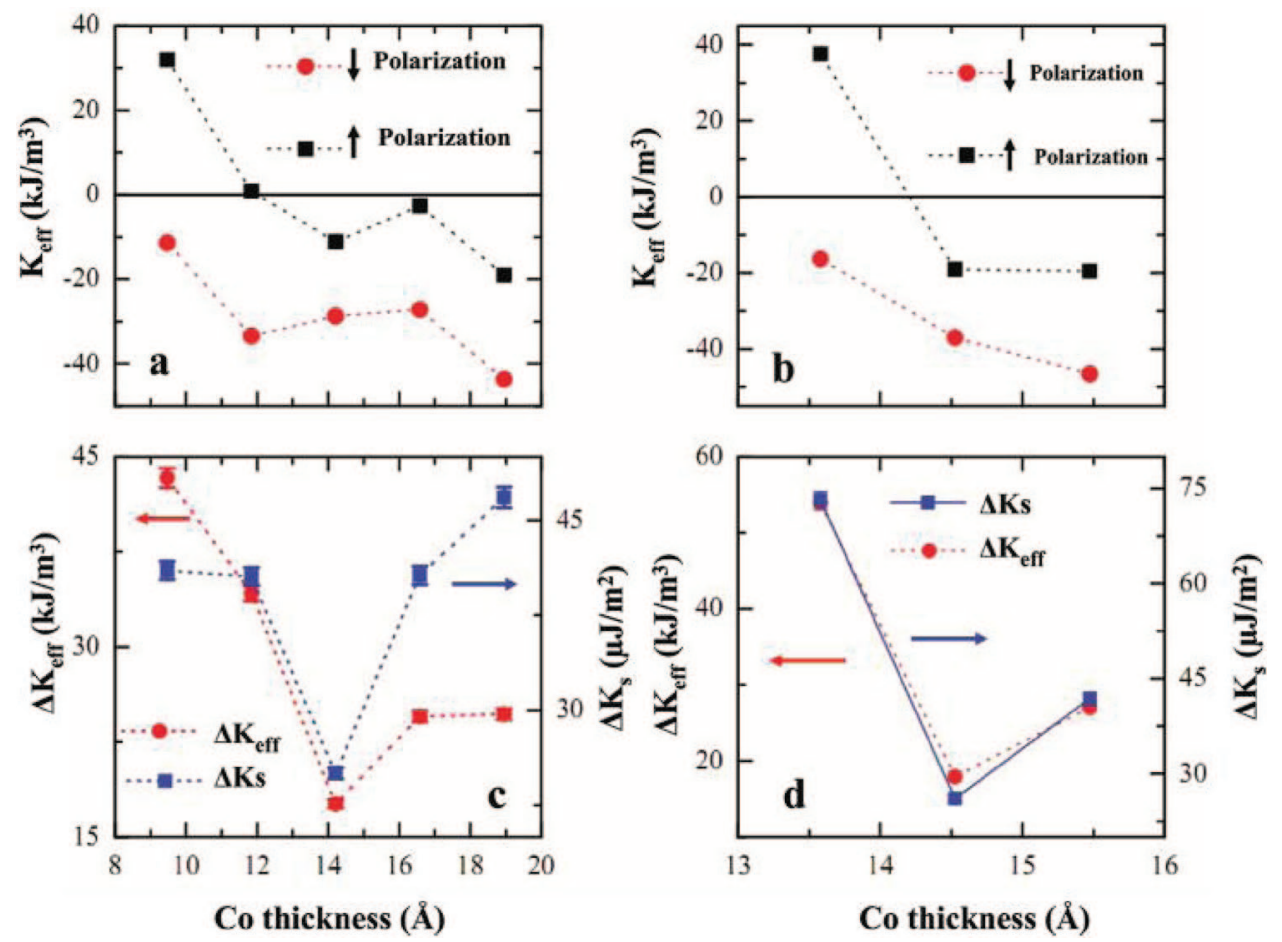

Figure 3. (a, b) Effective uniaxial anisotropy constants as obtained from the area method as a function of Co thickness for samples on a (a) Pd seed layer and (b) Pt seed layer. For the lowest thickness $(9.2 \AA) K_{\text {eff }}$ is positive for up polarization and negative for down polarization indicating a switching of the easy axis from out-of-plane to in-plane. At larger thickness, negative values of $K_{\text {eff }}$ result in in-plane magnetization. At the spin reorientation region for up polarization, the effective anisotropy is close to zero, in agreement with the $M_{\mathrm{r}} / M_{\mathrm{s}}$ data in Figure 2. (c, d) Changes in effective anisotropy $\Delta K_{\text {eff }}=K_{\text {eff }}(P \uparrow)-K_{\text {eff }}(P \downarrow)$ (red circle) and surface anisotropy $\Delta K_{\mathrm{s}}=t \Delta K_{\text {eff }}$ (blue square) resulting from switching of the ferroelectric polarization plotted as a function of Co thickness for samples on (c) a Pd seed layer and (d) a Pt seed layer.

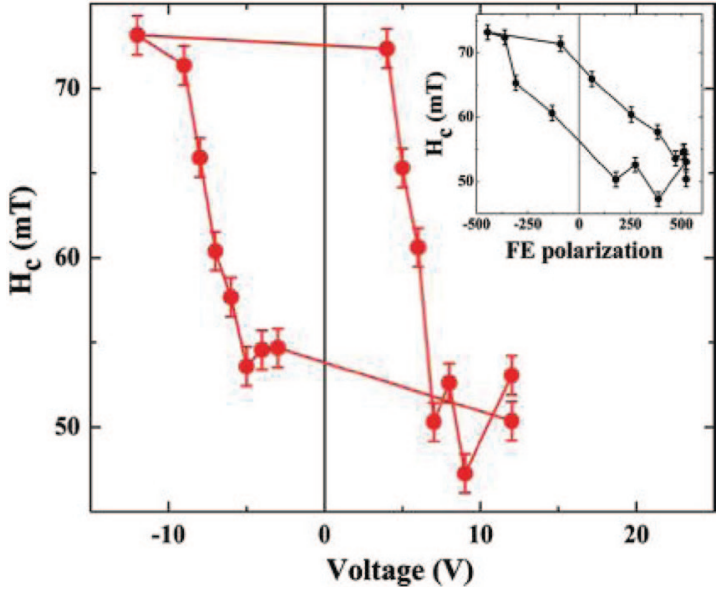

Figure 4. Out-of-plane magnetic coercivity, as a function of applied voltage, (and net ferroelectric polarization) measured at a Co thickness of $11.2 \AA$ at zero applied voltage, after the requisite voltage has been applied to change polarization. Coercivity measurements as a function of applied voltage show a shape similar to the ferroelectric polarization. Inset: out-of-plane coercivity as a function of ferroelectric polarization (as measured by the pyroelectric current). The polarization values corresponding to applied voltage were taken from a polarization hysteresis loop similar to that shown in Figure 1c.

The results of these experiments show that the changes in magnetic behavior cannot be attributed to volume effects. Symmetry considerations dictate that there should be no change in the in-plane strain in the ferroelectric film on polar- ization reversal and therefore no strain effects in the Co. (Any out-of-plane strain in the ferroelectric film would not have induced stress in the films, because the sample thickness was unconstrained.) Further, any residual strains in the polymer ferroelectric film are unlikely to perturb the Co, because of the much lower stiffness coefficient of the polymer.

The high electrical polarization charge, $0.1 \mathrm{C} / \mathrm{m}^{2}$, at the polymer surface contributes an appreciable electric field. Assuming a 10-15 A naturally occurring overlayer of $\mathrm{CoO},{ }^{27}$ the electric field penetrating the Co surface is calculated to be 8.74 $\times 10^{8} \mathrm{~V} / \mathrm{m}$ and extends into the metallic Co layer over a distance of $\sim 1.5 \AA$, altering the spin polarization over this depth because of unequal spin-up and spin-down screening charge densities. ${ }^{2,5}$ From our data the maximum change in the anisotropy due to the induced electric field is $\Delta K_{\text {eff }} / \Delta E=4.5 \times 10^{-5}$ $\mathrm{J} /\left(\mathrm{m}^{2} \mathrm{~V}\right)$. This may be compared to the much smaller experimentally obtained values of $2.1 \times 10^{-8} \mathrm{~J} /\left(\mathrm{m}^{2} \mathrm{~V}\right)$ found with a $\mathrm{Fe} / \mathrm{MgO}$ heterostructure, ${ }^{10}$ where the electric field was produced by applying relatively large applied voltages of $\pm 200 \mathrm{~V}$ and persisted only as long as the voltage was applied. Theoretical predictions of surface anisotropy changes ${ }^{1}$ in the transition metals of $\sim 10^{-14} \mathrm{~J} /(\mathrm{V} \mathrm{m})$ are very close to our experimentally observed value $\Delta K_{\mathrm{s}} / \Delta E \sim 6 \times 10^{-14} \mathrm{~J} /(\mathrm{V} \mathrm{m})$. These predicted large changes, however, assume extremely large applied electric fields of $1 \mathrm{~V} / \AA$, well above the breakdown field of oxides. In contrast, the magnetization changes seen in our system were obtained at low applied voltages (12 V) and persisted when the applied voltage was set to zero.

The effect of the ferroelectric polarization on the magnetic anisotropy energy is also apparent in an investigation of the magnetic switching behavior, as illustrated by Figure 5a. The two out-of-plane magnetization hysteresis loops, one for 

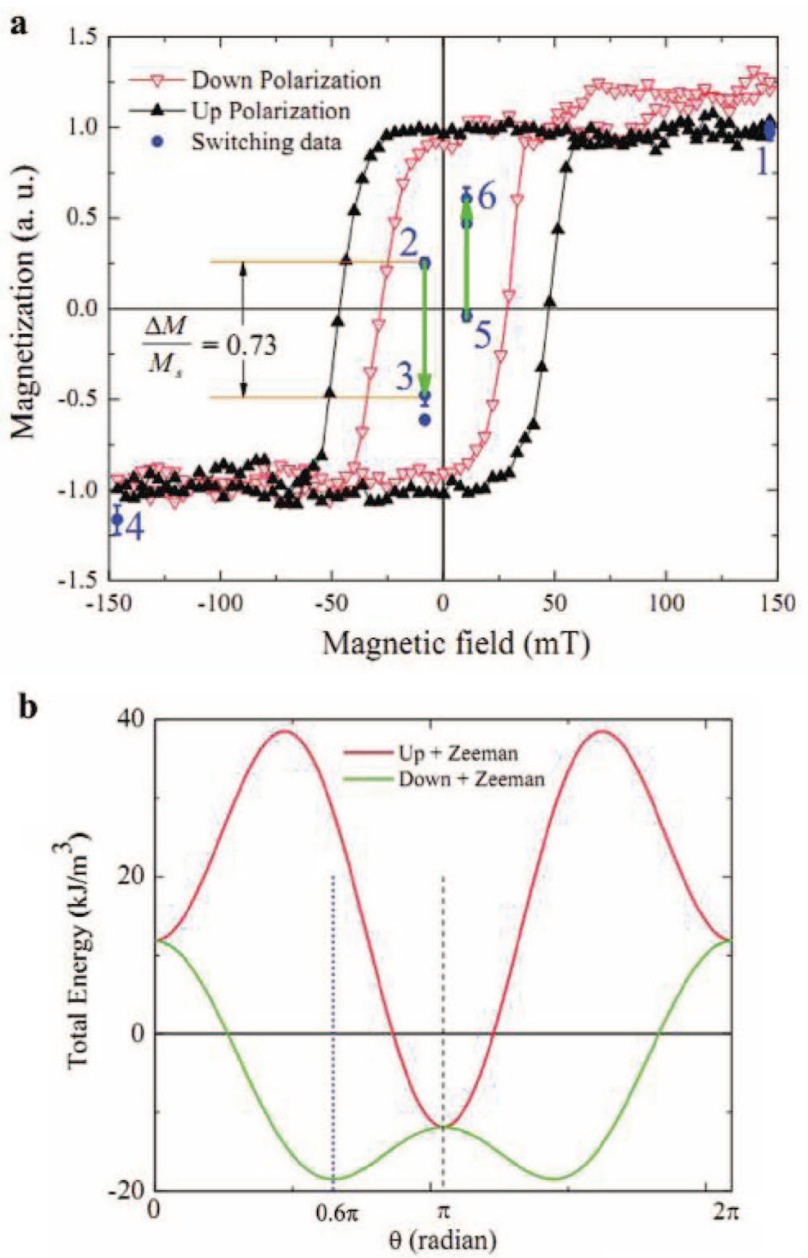

Figure 5. The red and black magnetic hysteresis loops for Co thickness of $8.8 \AA$ correspond to polarization pointing down and up, respectively. (a) Starting from positive magnetic saturation, with polarization pointing up, we reduce the magnetic field to $-8.3 \mathrm{mT}$ resulting in a lowering of the magnetization to point 2 . Switching the FE polarization to down results in a reduction of the magnetization to point 3 , due to the lowering of the energy barrier with the change in polarization. Point 3 is stable and changing the polarization does not switch it back to point 2 . Similarly after negative magnetic saturation and polarization up, we increase the field to 5, switch the FE polarization to down resulting in a change of the magnetization to point 6 . Switching back to up polarization does not alter state 6, which like 3 is stable to changes in the ferroelectric polarization. (b) Calculations of the magnetic free energy consisting of the magnetic anisotropy (which is altered by the direction of ferroelectric polarization) and Zeeman energies for the two polarization states. Note the lowering of the energy barrier when the polarization is switched from up to down and the minima of energy occurring at $\theta=0.6 \pi$.

polarization up and the other for polarization down, are shown for the Pt/Co film at a Co thickness of $8.8 \AA$. The ferroelectric film was polarized up and the magnetic film was saturated, resulting in the magnetization denoted as point 1 in Figure $5 \mathrm{a}$. On reducing the applied magnetic field to $-8.34 \mathrm{mT}$, the magnetization value dropped rapidly (a consequence of magnetic relaxation effects) to the state denoted by 2 in Figure 5a, where it was stable for an extended period of time. Switching the ferroelectric film to the opposite (down) polarization state resulted in an abrupt change of the magnetization to point 3 in the hysteresis loop (Figure 5a). Reversing the polarization to up had no effect on the magnetization. A similar sequence with polarization up, negative magnetic saturation, reduction of the field to +10.43 $\mathrm{mT}$ and switching of the polarization results in the magnetization switching from 5 to 6 . Both 2 to 3 and 5 to 6 are irreversible with electric field, requiring the presence of a magnetic field to reverse the magnetization state.

The irreversible polarization-induced switching results from the free-energy landscape of the magnetic state and its dependence on the polarization direction. The magnetic free energy in a uniaxial system with no magnetocrystalline anisotropy may be written as $U=K_{\text {eff }} \sin ^{2} \theta-\vec{M} \cdot \vec{H}$, where $K_{\text {eff }}$ is the effective anisotropy, which in our sample is dependent on the electric field, $\theta$ is the angle the magnetization makes with the normal to the film, and $M$ and $H$ are the saturation magnetization and applied magnetic field, respectively. Because we could not measure the in-plane magnetization for the $8.8 \AA$ film, we use the $K_{\text {eff }}$ values for the $13.5 \AA$ film, 37.6 and $-16.3 \mathrm{~kJ} / \mathrm{m}^{3}$ for up and down polarization states, respectively, to calculate the free energy curves shown in Figure 5b. For up polarization, at an applied field of $-8.3 \mathrm{mT}$, the free energy barrier to magnetization reversal prevents rotation of the magnetization into the field direction, and the magnetization is stable at $\theta=0$, corresponding to point 2 in Figure 5a. Switching to down polarization lowers the energy barrier with the minimum of energy occurring at $\theta=0.6 \pi$, i.e., the magnetization lies close to the in-plane direction at point 3 in Figure 5a. Because PMOKE measures only the perpendicular component of the magnetization, we expect to measure a value of $-0.36 M_{\mathrm{s}^{\prime}}$ close to the actual value of $-0.5 M_{\mathrm{s}^{\prime}}$ which was measured at point 3 . The irreversibility of this transition is due to the large energy barrier encountered in going from point 3 to point 2 (or from point 6 to point 5 ).

In conclusion, we have shown that the electric polarization state of a polymer ferroelectric thin film substantially alters the magnetic anisotropy of a thin film transition metal ferromagnet, changing the magnetization easy axis from out-of-plane to in-plane for sufficiently thin ferromagnetic films. This magnetization switching from out-of-plane to in-plane is achieved using an applied voltage of only $12 \mathrm{~V}$, is stable at remanence, and is irreversible with electric field alone. The change in magnetic anisotropy is proportional to the polarization of the polymer ferroelectric, and the effect is absent above the ferroelectric transition temperature. Moreover, this behavior is achieved in a device with relaxed constraints on the ferroelectric/ferromagnetic interface as exemplified by the ex situ growth of the ferroelectric in a water subphase and the naturally occurring oxide layer on the ferromagnet. Both of these confer distinct benefits in the realization of nonvolatile memory devices and are in stark contrast to the demanding fabrication requirements of oxide ferroelectric films. The changes in the surface anisotropy, $30-70 \mu \mathrm{J} / \mathrm{m}^{2}$, are an order of magnitude higher than those seen in previous experiments ${ }^{10}$ and the magnetoelectric anisotropy coupling coefficient, $\Delta K_{\text {eff }} / \Delta E$, is almost 3 orders of magnitude higher.

The substantial mismatch between the stiffness coefficients of the ferromagnet and the ferroelectric precludes strain effects and the weak interfacial coupling rules out atomic rearrangements at the interface. Hence we infer that this is purely an electric field effect, arising from the large surface charge density at the ferroelectric/feromagnet interface that results in a large electric field that will penetrate the metallic Co over a distance equal to the screening length. This alters the anisotropy energy barrier for magnetic switching allowing for electric control of magnetic switching at very low applied voltages of $\pm 12 \mathrm{~V}$, with the magnetic state remaining stable in the absence of applied voltage. 
Methods - The metallic bottom electrodes were sputtered through shadow masks $0.2 \mathrm{~mm}$ wide and $40 \mathrm{~mm}$ long on glass substrates at a base pressure of $3.7 \times 10^{-8}$ Torr. The Co layer with a wedge angle of $2.7 \times 10^{-6} \mathrm{deg}$ was deposited on a seed layer of either $\mathrm{Pd}$ or $\mathrm{Pt}(50 \mathrm{~nm})$. The deposition rates of $\mathrm{Pt}, \mathrm{Pd}$, and Co were $0.46,0.65$, and $0.216 \AA /$ s, respectively. The polymer ferroelectric films were deposited ex situ directly on the cobalt films by the Langmuir-Blodgett (LB) technique. The Langmuir layer was formed on an ultrapure water subphase using a $0.05 \%$ concentration of $\mathrm{P}(\mathrm{VDF}-\mathrm{TrFE})$ (70:30) in dimethyl sulfoxide. The layer was then compressed to a surface pressure of $5 \mathrm{mN} / \mathrm{m}$ at a temperature of $25^{\circ} \mathrm{C}$ and deposited onto the substrate using horizontal Langmuir-Blodgett deposition, with the film thickness determined by the number of transferred monolayers (ML). The LB deposited copolymers of 70\% VDF with $30 \%$ of TrFE, [P(VDF-TrFE) 70:30] are highly crystalline, excellent ferroelectrics with a saturation polarization of up to $0.1 \mathrm{C} /$ $\mathrm{m}^{2}$. The sample was then annealed at $130{ }^{\circ} \mathrm{C}$ for an hour in air at a ramp rate of $1.6^{\circ} \mathrm{C} / \mathrm{min}$ for both heating and cooling to increase the crystallinity of the polymer film. The $0.2 \mathrm{~mm}$ wide top $\mathrm{Al}$ electrode was deposited on top of the polymer film by evaporation at a deposition rate of $1.2 \AA / \mathrm{s}$. The top and bottom electrodes form a crossed pattern and defining an overlapping electrode area of $0.04 \mathrm{~mm}^{2}$. Copper wires were attached to the top and bottom electrodes with silver epoxy, allowing for pyroelectric measurements and enabling polarization switching. The ferroelectric properties of the polymer film were characterized by pyroelectric measurements using the Chynoweth method in which the sample temperature was modulated using a $3 \mathrm{~mW}$ laser beam at a chopper frequency of $2 \mathrm{kHz}$, and the pyroelectric current was measured by a lock-in amplifier with a time constant of $1 \mathrm{~s}$. The magnetic layers of the samples have been characterized using the Magneto-optical Kerr effect (MOKE) using longitudinal MOKE for in-plane measurements and polar MOKE for out-of-plane measurements. The magnetic anisotropy was calculated using the area method (see Figure S3 in the Supporting Information), where we assume the bulk magnetization value for Co.

Acknowledgments - This work was supported by the National Science Foundation through the Materials Research Science and Engineering Center program under Grant No. DMR-0820521.

Supporting Information follows the References.

\section{References}

1. Duan, C. G. Surface magnetoelectric effect in ferromagnetic metal films Phys. Rev. Lett. 2008, 101, 137201

2. Zhang, S. Spin-Dependent Surface Screening in Ferromagnets and Magnetic Tunnel Junctions Phys. Rev. Lett. 1999, 83, 640

3. Duan, C. G. Tailoring magnetic anisotropy at the ferromagnetic/ferroelectric interface Appl. Phys. Lett. 2008, 92, 122905

4. Nakamura, K. Origin of electric-field-induced modification of magnetocrystalline anisotropy at Fe(100) surfaces: Mechanism of dipole formation from first principle Phys. Rev. B 2009, 80, 172402

5. Cai, T. Magnetoelectric coupling and electric control of magnetization in ferromagnet/ferroelectric/normal-metal superlattices Phys. Rev. B 2009, 80, 140415(R)

6. Rondinelli, J. M.; Stengel, M.; Spaldin, N. A. Carrier-mediated magnetoelectricity in complex oxide heterostructures Nat. Nanotechnol. 2008, 3, 46
7. Chiba, D.; Yamanouchi, M.; Matsukura, F.; Ohno, H. Electrical manipulation of magnetization reversal in a ferromagnetic semiconductor Science 2003, 301, 943

8. Ohno, H. Electric-field control of ferromagnetism Nature 2000, 408, 944

9. Weisheit, M. Electric Field-Induced Modification of Magnetism in Thin-Film Ferromagnets Science 2007, 315, 349

10. Maruyama, T. Large voltage-induced magnetic anisotropy change in a few atomic layers of iron Nat. Nanotechnol. 2009, 4,158

11. Shiota, Y. Voltage-assisted magnetization switching in ultrathin $\mathrm{Fe}_{80} \mathrm{Co}_{20}$ alloy layers Appl. Phys. Express 2009, 2, 063001

12. Endo, M.; Kanai, S.; Ikeda, S.; Matsukura, F.; Ohno, H. Electric-field effects on thickness dependent magnetic anisotropy of sputtered $\mathrm{MgO} / \mathrm{Co}_{40} \mathrm{Fe}_{40} \mathrm{~B}_{20} /$ Ta structures Appl. Phys. Lett. 2010, 96, 212503

13. Sahoo, S. Ferroelectric control of magnetism in $\mathrm{BaTiO}_{3} / \mathrm{Fe}$ heterostructures via interface strain coupling Phys. Rev. B 2007, 76, 092108

14. Spaldin, N. A.; Ramesh, R. Electric-field control of magnetism in complex oxide thin films Science 2008, 33, 1047

15. Duan, C. G.; Jaswal, S. S.; Tsymbal, E. Y. Predicted Magnetoelectric effect in $\mathrm{Fe} / \mathrm{BaTiO}_{3}$ Multilayers: Ferroelectric Control of Magnetism Phys. Rev. Lett. 2006, 97, 047201

16. Ducharme, S.; Palto, S. P.; Fridkin, V. M. Ferroelectric and Dielectric Thin Films; Nalwa, H. S., Ed.; Academic Press: San Diego, 2002; Vol. 3, pp 545- 591.

17. Palto, S. Ferroelectric Langmuir-Blodgett films Ferroelectr., Lett. Sect. 1995, 19, 65

18. Carcia, P. F. Perpendicular magnetic anisotropy in Pd/Co and Pt/Co thin-film layered structures J. Appl. Phys. 1988, 63, 5066

19. Lee, J. W.; Jeong, J. R.; Shin, S. C.; Kim, J.; Kim, S. K. Spin -reorientation transitions in ultrathin Co films on $\mathrm{Pt}(111)$ and Pd(111) single-crystal substrates Phys. Rev. B 2002, 66, 172409

20. Sorokin, A. V.; Fridkin, V. M.; Ducharme, S. Pyroelectric study of polarization switching in Langmuir-Blodgett films of poly(vinylidene fluoride trifluoroethylene) J. Appl. Phys. 2005, 98, 044107

21. Lopez, J. M.; Sun, Y.; Burton, J. D.; Tsymbal, E. Y.; Velev, J. $P$. Organic multiferroic tunnel junctions with ferroelectric poly(vinylidene difluoride) barriers Nano Lett. 2011, 11, 599

22. Blinov, L. M.; Fridkin, V. M.; Palto, S. P.; Sorokin, A. V.; Yudin, S. G. Thickness Dependence of Switching for Ferroelectric Langmuir Films Thin Solid Films 1996, 284-85, 474

23. Sorokin, A. V.; Ducharme, S.; Fridkin, V. M. Pyroelectric study of a polarization switching in Langmuir-Blodgett films of poly(vinylidene fluoride - trifluoroethylene) J. Appl. Phys. 2005, 98, 044107

24. Johnson, M. T.; Bloemen, P. J. H.; den Broeder, F. J. A.; de Vries, J. J. Magnetic anisotropy in metallic multilayers Rep. Prog. Phys. 1996, 59, 1409

25. Allenspach, R.; Stampanoni, M.; Bischof, A. Magnetic domains in thin epitaxial $\mathrm{Co} / \mathrm{Au}(111)$ films Phys. Rev. Lett. 1990, 65, 3344

26. Rodriguez, B. J.; Jesse, S.; Kalinin, S. V.; Kim, J.; Ducharme, S. Nanoscale polarization manipulation and imaging of ferroelectric Langmuir-Blodgett polymer films Appl. Phys. Lett. 2007, 90, 122904

27. Maat, S.; Takano, K.; Parkin, S. S. P.; Fullerton, E. E. Perpendicular Exchange Bias of Co/Pt Multilayers Phys. Rev. Lett. 2001, 87,087202 


\section{Supporting Information}

\section{The shift in the spin reorientation transition of $\mathrm{Co}$}

Deposition of the FE layer results in a substantial shift in the position of the spin reorientation transition (SRT) on the Co wedge, as shown in figure S1 (a). Measurements of both in-plane and out-of-plane MOKE and the corresponding $\mathrm{M}_{\mathrm{r}} / \mathrm{M}_{\mathrm{s}}$ values indicate that the SRT shifts from a thickness of approximately $10 \AA$ without the FE polymer to $13 \AA$ after the polymer film has been deposited and annealed. This shift is attributed to interfacial chemistry at the metal polymer interface. Cobalt has been shown to be particularly reactive with polymers, ${ }^{1}$ with the formation of carboxylates, $\mathrm{Co}(\mathrm{OH})_{2}$ as well as clusters of metallic Co. Cobalt films exposed to ambient conditions, as was this sample, form oxides and hydroxides, ${ }^{2}$ with rates of formation dependent on temperature and time, further complicating the issue. A series of experiments to explore the origin of the dramatic shift in the position of the SRT were carried out on a Co wedge (identical to the $\mathrm{Pt} / \mathrm{Co}$ wedge) with thickness ranging from $4 \AA$ to $17 \AA$ using the magneto-optical Kerr effect (MOKE) to pinpoint changes in the SRT (see supporting figure S1 (b)). A set of four MOKE measurements were made in sequence: (i) on the bare Co surface after removal from the deposition chamber (ii) after immersion in ultrapure water, as would occur for the LB deposition process, effected by dipping the sample into a clean LB trough (iii) after deposition of the PVDF and (iv) after annealing of the PVDF film. The SRT remained unaffected by the first three steps, shifting dramatically from $8 \AA$ to $12 \AA$ only after annealing of the PVDF film. We attribute this change to interfacial chemical changes that are accelerated by the $130{ }^{\circ} \mathrm{C}$ annealing temperature, rather than to electric field effects from the polarized ferroelectric. This is because, although annealing does increase the net polarization of PVDF, ${ }^{3}$ magnetic hysteresis loops for the out of-plane easy axis direction recorded above the Curie temperature of the 
ferroelectric polymer showed no change from the room-temperature measurements (see supporting figure 2).

\section{The effect of temperature}

In order to confirm the central role of the ferroelectric polarization in the magnetic effects observed, the Pt/Co sample was heated to $119.4{ }^{\circ} \mathrm{C}$, well above the ferroelectric-paraelectric phase transition temperature of $107^{\circ} \mathrm{C} .{ }^{4}$ The out-of plane MOKE measurement at a Co thickness of $11.2 \AA$ corresponding to two different applied voltage of $+12 \mathrm{~V}$ and $-12 \mathrm{~V}$, the same voltage used previously to polarize the FE layer is shown in figure S2 (a). There was no significant effect of the sign of the applied voltage in the magnetic coercivity, which was measured at zero voltage in both cases. At this temperature, the ferroelectric film does not remain polarized without a voltage. On cooling back down to room temperature, the polymer film was confirmed to be ferroelectric through polarization hysteresis measurement (see figure S2 (b)) and again the out-of plane MOKE measurements in the two different polarization states, up $(-12 \mathrm{~V})$ and down $(+12 \mathrm{~V})$, showed the change in coercivity of $8.9 \mathrm{mT}$ as shown in figure $\mathrm{S} 3$ (c). The much lower coercivity of the magnetic film at high temperature (compare figure S2 (a) and (c)) is a feature common to ferromagnets, and not necessarily due to the loss of ferroelectric polarization in the polymer film. To test this hypothesis with our Co films, a new sample of Pd $(50 \mathrm{~nm}) / \mathrm{Co}(1.4 \mathrm{~nm})$ was made. The MOKE measurement from this sample (Figure S3 (d)) also exhibits a decrease in coercivity by $5.1 \mathrm{mT}$ from room temperature to at $119.4{ }^{\circ} \mathrm{C}$, the same amount observed with the cobalt-polymer heterostructure (figure S2 (a)). 


\section{Supporting References}

${ }^{1}$ Leidheiser, H. \& Deck, P. D. Chemistry of the Metal-Polymer Interfacial Region. Science 241, $1176(1988)$.

${ }^{2}$ Tompkins, H.G. \& Augis, J.A. The oxidation of cobalt in air from room temperature to $467^{\circ} \mathrm{C}$. Oxidation of metals, 16, 355 (1981).

${ }^{3}$ Bai, M. Poulsen, M. \& Ducharme, S. Effects of annealing conditions on ferroelectric nanomesa self-assembly. J. Phys.: Condens. Matter 18, 7383 (2006).

${ }^{4}$ Poulsen, M. Sorokin, A. V. Adenwalla, S. Ducharme, S. \& Fridkin, V. M., Effects of an external electric field on the ferroelectric-paraelectric phase transition in polyvinylidene fluoridetrifluoroethylene copolymer Langmuir-Blodgett films. J. Appl. Phys. 103, 034116 (2008).

\section{Supporting figure captions}

Fig. S1. (a) $M_{r} / M_{s}$ data for both in-plane and out-of plane magnetization loops before and after the growth of the ferroelectric P(VDF-TrFE) 70:30 layer. i) in-plane data without FE layer (solid black) ii) out- of plane data without FE layer (open black) iii) in-plane data with FE layer (solid red) iv) out-of plane data with FE layer (open red) for the magnetization loop measurement. Clearly there is a shift in the transition region to the thicker side of the Co film as shown by the arrow from the green shaded region to the gray shaded region. (b) A set of four sequential MOKE measurements were made on an identical Pt/Co wedge sample with thickness ranging from $4 \AA$ to $17 \AA$ : (i) on the bare Co surface (ii) after immersion into water (iii) after deposition of the PVDF and (iv) after annealing of the PVDF film. The SRT transition remained unaffected by the first three steps, shifting dramatically from $8 \AA$ to $12 \AA$ only after annealing of the PVDF film. 
Fig. S2. (a) At $119.4{ }^{\circ} \mathrm{C}$, well above the ferroelectric-paraelectric phase transition temperature there is no change in coercivity. (b) and (c) are room temperature measurements of the b, ferroelectric loop and c, magnetization loop indicating that the sample is stable. (d) The lower coercivity at high temperature is an expected feature. A Pd/Co sample without the FE layer shows a similar temperature dependence of the coercivity.

Fig. S3. Schematics of the magnetic anisotropy calculation using the area method. The black and red magnetization curve gives the area in the first quadrant of the magnetic hysteresis loop for the easy and hard axis directions respectively. If we assume that domain losses are identical for both in-plane and out-of-plane orientations, the difference between these two areas (shown in green) gives the effective anisotropy energy, which contains both the surface and volume anisotropy energies. 

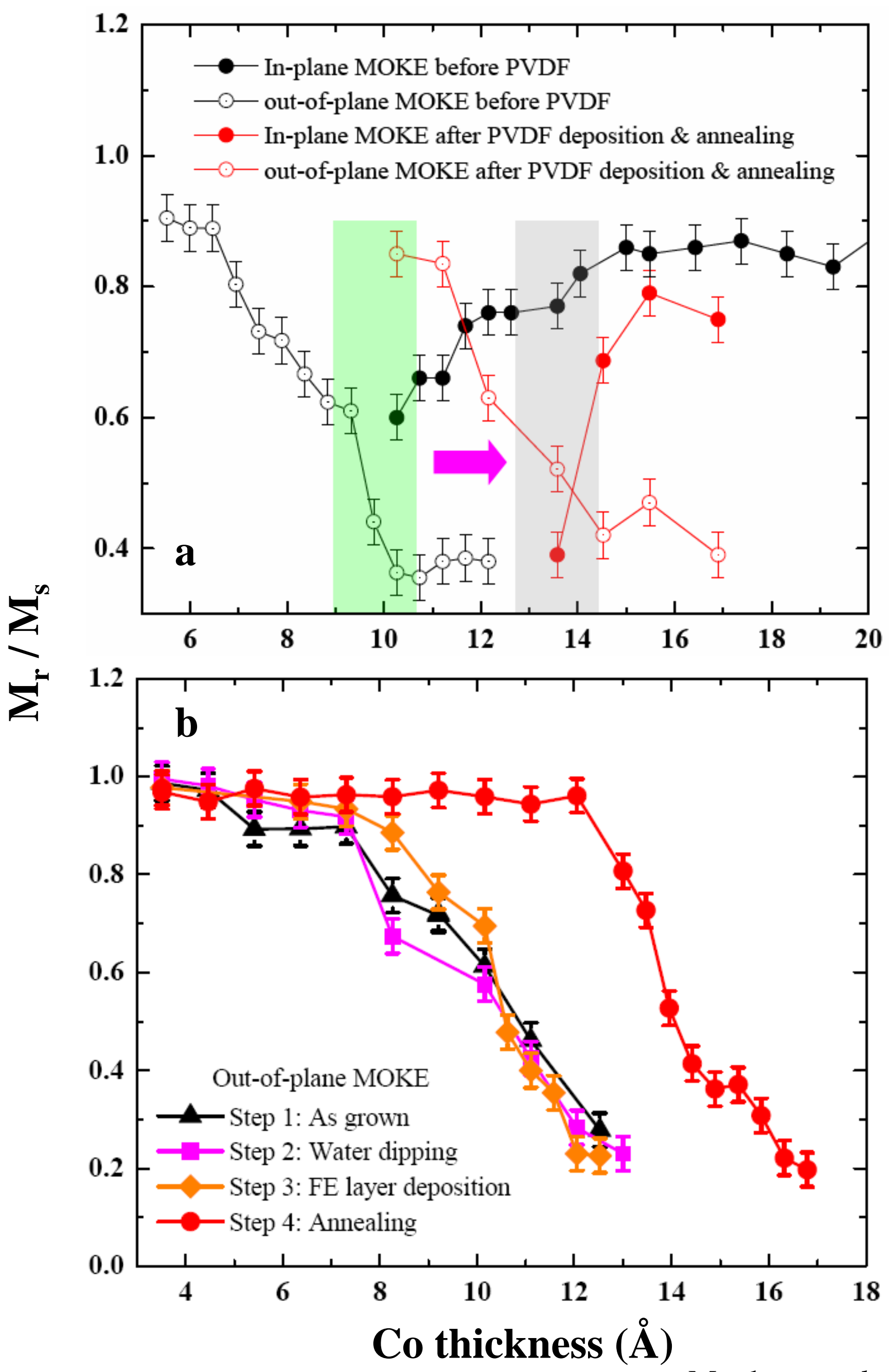

Mardana et al., Figure S1 

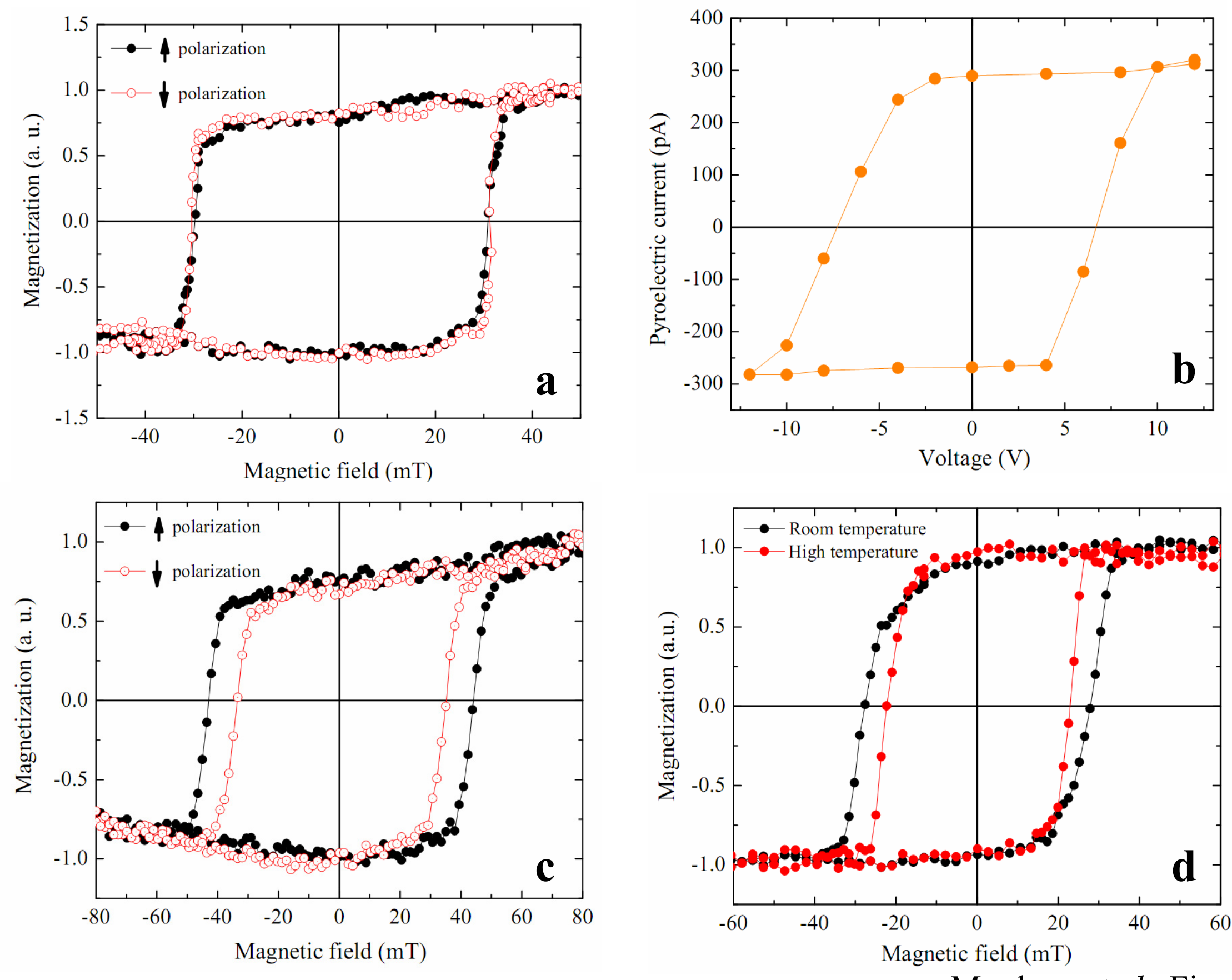

Mardana et al., Figure S2 


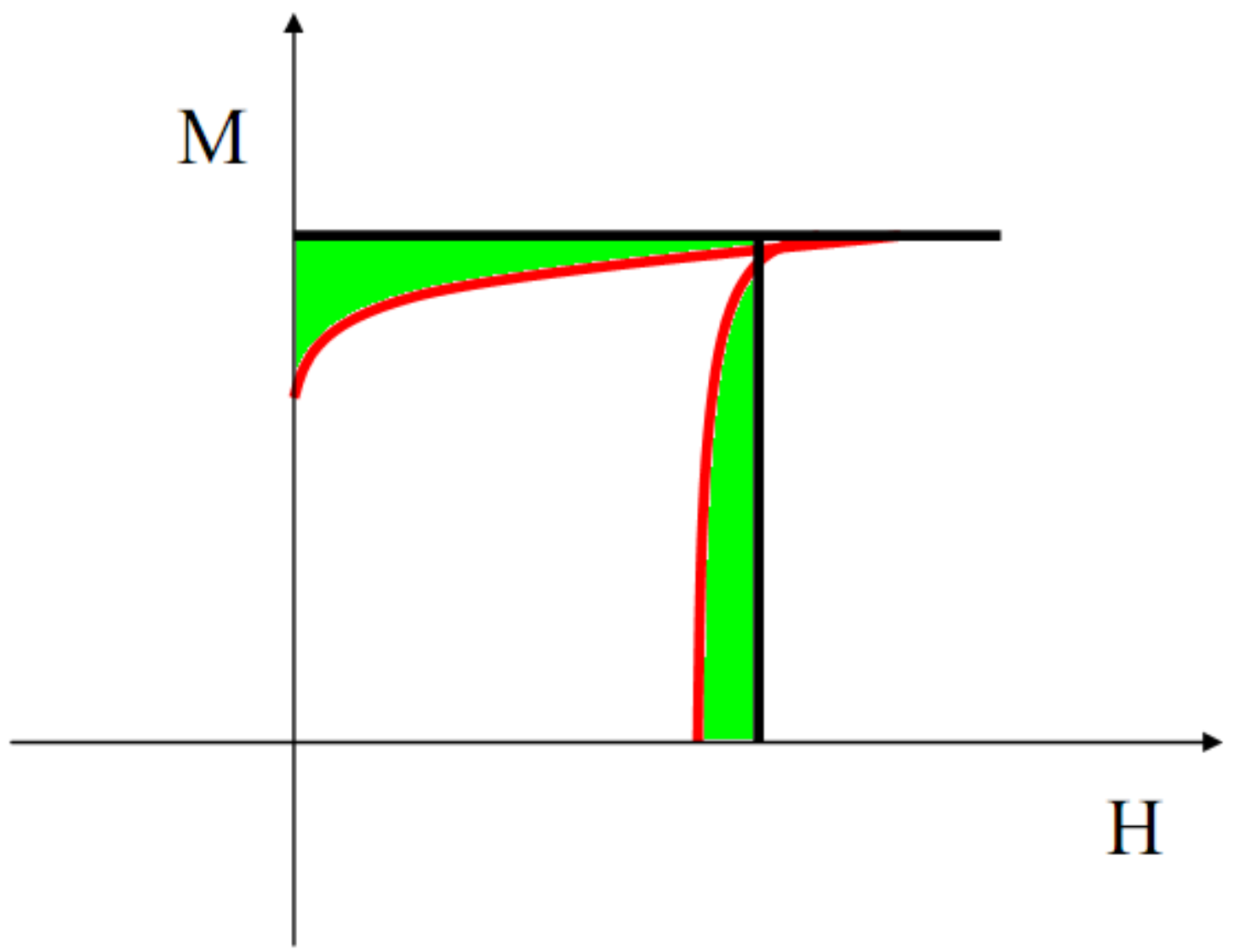

Mardana et al., Figure S3 